\title{
Radiative and non-radiative electron capture from carbon atoms by relativistic helium ions
}

\author{
A. Gójska ${ }^{1, a}$, D. Chmielewska ${ }^{1}$, P. Rymuza ${ }^{1}$, J. Rzadkiewicz ${ }^{1}$, Z. Sujkowski ${ }^{1}$, T. Adachi ${ }^{2}$, H. Fujita $^{2}$, Y. Fujita $^{2}$, \\ Y. Shimbara ${ }^{2}$, K. Hara ${ }^{3}$, Y. Shimizu ${ }^{3}$, H.P. Yoshida ${ }^{3}$, Y. Haruyama ${ }^{4}$, J. Kamiya ${ }^{5}$, H. Ogawa ${ }^{6}$, M. Saito ${ }^{7}$, and \\ M. Tanaka ${ }^{8}$ \\ 1 The Andrzej Soltan Institute for Nuclear Studies, 05-400 Otwock - Świerk, Poland \\ 2 Department of Physics, Osaka University, Toyonaka, Osaka 560-0043, Japan \\ 3 Research Center for Nuclear Physics, Osaka University, Ibaraki, Osaka, 567-0047, Japan \\ 4 Laboratory of Applied Physics, Kyoto Prefectural University, 1 Hangicho, Shimogamo, Sakyo-ku, Kyoto, 606-8522, Japan \\ 5 IPNS (Institute of Particle and Nuclear Studies), KEK (High Energy Accelerator Research Organization), Oho 1, Tsukuba, \\ Ibaraki 305-0801, Japan \\ 6 Department of Physics, Nara Women's University, Kitauoya-nishimachi, Nara 630-8506, Japan \\ 7 Kyoto Prefectural University, Kyoto 606-8522, Japan \\ 8 Kobe Tokiwa Jr. College, Nagata, Kobe, 653-0824, Japan
}

Received: 11 July 2005 /

Published online: 29 March 2006 - (C) Società Italiana di Fisica / Springer-Verlag 2006

\begin{abstract}
Interaction of radiation with helium atoms and/or ions is of interest in various astrophysical applications. The reverse reactions of fast $150 \mathrm{MeV} / \mathrm{amu}{ }^{3} \mathrm{He}^{++}$ions with solid $\mathrm{C}$ targets have been studied at the isochronous cyclotron of the RCNP in Osaka. The singly ionized helium ions resulting from capture of the target electrons to the projectile were observed with the use of large magnetic spectrograph, Grand Raiden, set at $\theta=0^{\circ}$ with respect to the beam. The yield ratios of singly-to-doubly ionized helium ions emerging from thin carbon foils, $\mathrm{He}^{+} / \mathrm{He}^{++}$, have been measured as a function of the foil thickness. Extrapolating the results to zero target thickness permits to determine the cross section values separately for electron stripping from ${ }^{3} \mathrm{He}^{+}$ions and for electron capture to ${ }^{3} \mathrm{He}^{++}$ions. The radiative and nonradiative contributions to the capture cross section were determined in a separate experiment in which the $84 \mathrm{keV}$ (C.M.) photons were observed in coincidence with the $\mathrm{He}^{+}$ejectiles. The results are compared with theoretical predictions. Need for improved calculations is noted for all the three processes involved, i.e. for the radiative and non radiative electron capture as well as for the electron stripping.
\end{abstract}

PACS. 25.40.Lw Radiative capture $-34.70 .+\mathrm{e}$ Charge transfer

\section{Introduction}

The main processes occurring during the passage of ions through matter are the electron capture from the target to the vacant states of the projectile and the ionization (stripping) of the bound electrons from the passing ion (see [1] for a general review). Cross sections for these processes depend sharply on the velocity of the projectile as well as on the atomic number of the projectile and of the target. There seems to be no satisfactory theoretical description of the ionization process for fast projectiles. Rather crude approximations are used, applicable in limited energy and $Z_{T}, Z_{P}$ ranges, where $Z_{T}, Z_{P}$ are the atomic numbers of the target atom and of the projectile, respectively. The classical Bohr theory [2] for low $Z_{T}$ and not very fast ions

\footnotetext{
${ }^{a}$ e-mail: gojska@ipj.gov.pl
}

predicts:

$$
\sigma_{S T R I P} \propto \frac{Z_{T}^{2}}{Z_{P}^{2} v^{2}},
$$

where $\sigma_{S T R I P}$ is the stripping cross section, $v$ is the projectile velocity.

The ionization cross section for fast ions interacting with multi electron target atoms requires more precise theory. The quantum description of the stripping process was given by Gillespie [3,4]:

$$
\sigma_{S T R I P} \propto \frac{I}{v^{2}}
$$

where $I$ is the collision strength. A phenomenological expression for $I\left(Z_{T}\right)$ can be found in [5].

Two very different mechanisms contribute to the electron capture: the radiative one, in which the excess energy 
is carried away by a photon, and the non-radiative one, NREC. Because of the conservation laws the latter process can occur only for the capture of electrons bound in the target atom. The competing radiative transfer of bound atomic electrons is referred to as the Radiative Recombination, RR, while the capture of free electrons is called the Radiative Electron Capture, REC. The REC process can be considered as the time-reversed photoelectric effect on the partly ionized projectile atom. The REC cross section, $\sigma_{R E C}$, grows quickly with $Z_{P}$ and decreases rapidly with $v$. For high but non-relativistic projectile energy:

$$
\sigma_{R E C} \propto \frac{Z_{T} Z_{P}^{4.5}}{v^{5}}
$$

In contrast, the NREC process occurs mainly at the velocity matching condition $v \approx v_{e}$, where $v_{e}$ is the velocity of the captured electron, bound in the target atom. For $v \gg v_{e}$ :

$$
\sigma_{N R E C} \propto \frac{Z_{T}^{5} Z_{P}^{5}}{v^{11}} .
$$

A simple approach to describe the non-radiative electron capture cross section is the Oppenheimer-BrinkmannKramer (OBK) theory [1]. The screening corrections are included in $[6,7]$, while the relativistic effects are described in [1]. Because of the sharp dependence of electron capture cross section on velocity as well as on $Z_{P}$ the measurements for fast light ions are difficult and require very refined techniques. Recent experimental information on interaction of fast helium ions with various gaseous and solid targets can be found in $[8,9]$. The authors have measured the stripping and the capture cross section for ${ }^{3} \mathrm{He}$ ions with energies up to $43.4 \mathrm{MeV} / \mathrm{amu}$. The $\sigma_{C A P} / \sigma_{S T R I P}$ ratios were measured for a number of thick targets in [5]. The present work extends this information to much higher ${ }^{3} \mathrm{He}$ energy, $150 \mathrm{MeV} / \mathrm{amu}$, for $Z_{T}=6$. This is the first measurement for such high-energy light projectile. Preliminary results were published in $[10,11]$. Similar measurements for $Z_{T}=79$ are described in [12]. The present results are compared with theoretical capture and stripping cross sections.

\section{Experiment}

\subsection{Total capture and stripping cross sections}

There is a huge difference in the magnitude of stripping and capture cross sections for a fast light ion traversing a solid. As a result, the electron captured by the ion in one collision is very quickly lost in the subsequent one. In effect, the charge state of the ion reaches the equilibrium conditions in very thin layers of the solid. In order to determine the interaction cross sections the target thickness used has to be significantly smaller than the equilibrium thickness. This is of the order of $100 \mu \mathrm{g} / \mathrm{cm}^{2}$ for solid carbon. A method to determine the total capture as well as the stripping cross section is to carry out an extrapolation experiment in which the yield ratio, $Y\left({ }^{3} \mathrm{He}^{+}\right) / Y\left({ }^{3} \mathrm{He}^{++}\right)$, of the singly-to-doubly ionized He ions emerging from the

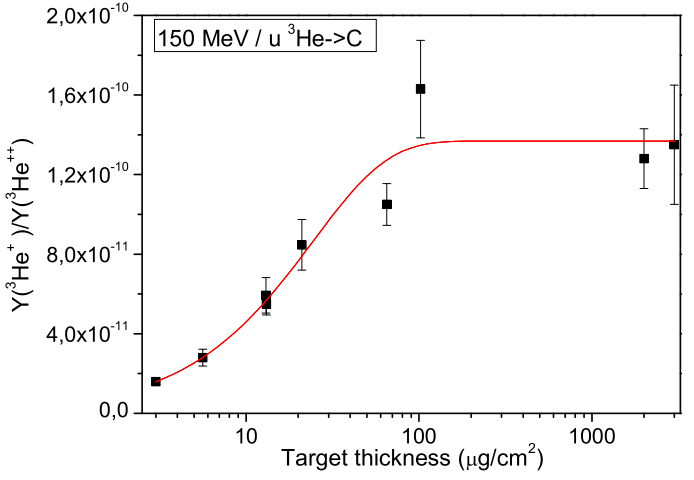

Fig. 1. The measured $Y\left({ }^{3} \mathrm{He}^{+}\right) / Y\left({ }^{3} \mathrm{He}^{++}\right)$yield ratio as a function of target thickness for $\mathrm{C}$ target. The errors are statistical only. The curve is a fit of eq. (6) to the data.

target is measured as a function of the target thickness. For thicknesses larger than the saturation value, $x_{\text {sat }}$, this ratio is equal to the cross section ratio [5]:

$$
\frac{Y\left({ }^{3} \mathrm{He}^{+}\right)}{Y\left({ }^{3} \mathrm{He}^{++}\right)}\left(x>x_{\text {sat }}\right)=\frac{\sigma_{C A P}}{\sigma_{S T R I P}} .
$$

Extrapolating the yield ratio to zero target thickness permits to separately determine the respective cross section values.

The ${ }^{3} \mathrm{He}^{++}$beam has been accelerated to $150 \mathrm{MeV} / \mathrm{amu}$ in the AVP cyclotron at the Research Center for Nuclear Physics (RCNP) in Osaka. The experimental set-up was identical to that used to study the $\left({ }^{3} \mathrm{He}, \mathrm{t}\right)$ nuclear charge exchange reactions [13]. The singly ionized ${ }^{3} \mathrm{He}^{+}$ions were detected together with tritons in the focal plane of the magnetic spectrometer Grand Raiden, set at $0^{\circ}$ with respect to the beam. The ${ }^{3} \mathrm{He}^{++}$beam was fully intercepted by a Faraday cup placed in the first dipole magnet of the spectrometer. The intensity ratio of singly-to-doubly ionized helium ions leaving the carbon target was measured as a function of the target thickness (fig. 1). Carbon targets: 3, 5.6, 13, $13.1,21,65,102,2000,3000 \mu \mathrm{g} / \mathrm{cm}^{2}$ thick were used. The errors in thicknesses were estimated to be between 5 and $10 \%$. Two sets of data were obtained under largely changed spectrograph settings used in the singles and coincidence experiments. A very satisfactory matching of these sets is observed. This builds up confidence in the proper reduction of possible systematic errors. The capture and stripping cross sections can be determined by fitting the simple function to the measured yield ratio versus target thickness:

$$
\frac{Y\left({ }^{3} \mathrm{He}^{+}\right)}{Y\left({ }^{3} \mathrm{He}^{++}\right)}=a[1-\exp (-b x)],
$$

where $a=\frac{\sigma_{C A P}}{\sigma_{S T R I P}+\sigma_{C A P}} \sim \frac{\sigma_{C A P}}{\sigma_{S T R I P}}$ cross section ratio, $b=\sigma_{S T R I P}+\sigma_{C A P} \sim \sigma_{S T R I P}$, and $x$ denotes the target thickness in $\mu \mathrm{g} / \mathrm{cm}^{2}$ multiplied by the number density of the target atoms. The implicit assumption for eqs. (5) and (6) is that the change in the ${ }^{3} \mathrm{He}^{++}$intensity after penetrating the target foil is negligible. 

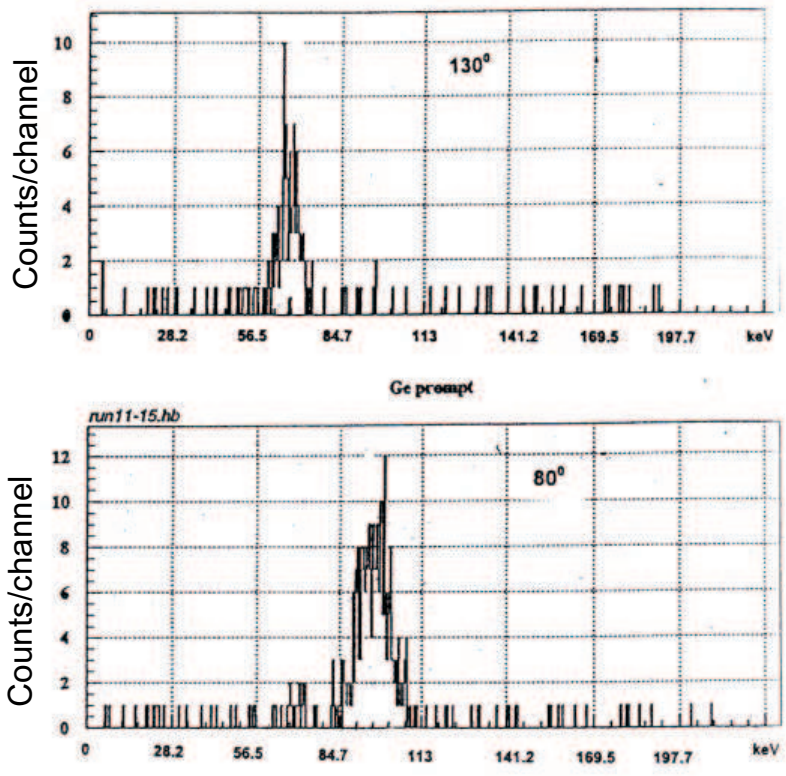

Fig. 2. The coincidence REC photon spectra.

\subsection{Coincidence experiment}

The extrapolation experiment measures the total electron capture cross section which is the sum of the non-radiative and radiative components

$$
\sigma_{C A P}=\sigma_{R E C}+\sigma_{N R E C} .
$$

The relative contributions of these components can be determined in a coincidence experiment in which the $\mathrm{He}^{+}$ ejectiles are registered in coincidence with the REC photons. Neglecting the electron binding energy in helium ion the energy of these photons corresponds to the energy of electrons in the centre-of-mass system of the projectile. The REC photons were measured in coincidence with ${ }^{3} \mathrm{He}^{+}$ions by the Ge detectors set at $\theta=80^{\circ}$ and $\theta=130^{\circ}$ with respect to the beam. The centre-of-mass energy is $E_{R E C}=84 \mathrm{keV}$. Figure 2 shows the measured spectra. The REC photons are strongly anisotropic $[14,15]$. In order to correct for this effect the coincidence yields were analysed according to the formula:

$$
\frac{\sigma_{R E C}}{\sigma_{t o t}}=\frac{N(R E C)}{(3 / 2) \varepsilon \sin ^{2} \theta N\left({ }^{3} \mathrm{He}^{++}\right)}
$$

where $Y(R E C)$ is the coincidence photon yield, $Y\left({ }^{3} \mathrm{He}^{++}\right)$ is the beam intensity, $\theta$ is the angle between the direction of observation and the direction of the beam and $\varepsilon$ is the efficiency of the photon detection.

\section{Results and discussion}

The measured values for the three cross sections are:

$\sigma_{S T R I P}=(821 \pm 60) \mathrm{kb}$,

$\sigma_{R E C}=(64 \pm 12) \mu \mathrm{b}$,

$\sigma_{N R E C}=(47 \pm 8) \mu \mathrm{b}$

These values are compared below with the respective theoretical predictions.
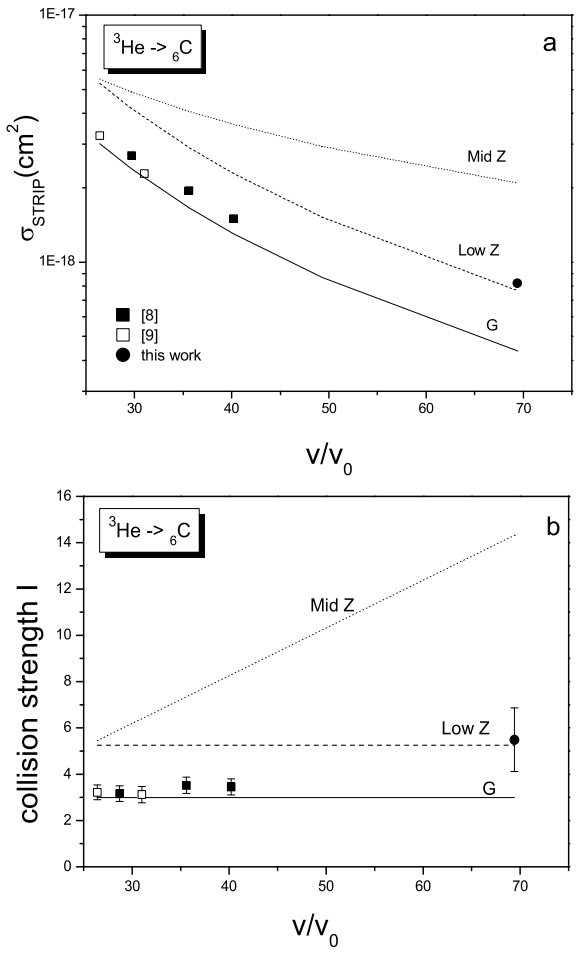

Fig. 3. The experimental (points) and calculated electron stripping cross section (a) and collision strength (b) as a function of ion velocity, in units of $v_{0}=2.19 \cdot 10^{8} \mathrm{~cm} / \mathrm{s}$. The solid line is Gillespie, the dotted and dashed lines are Bohr, approximations for medium- and low- $Z$ targets, respectively.

\subsection{Electron stripping from $\mathrm{He}^{+}$ions}

The existing data on the stripping cross section in the energy range $17.3-43.3 \mathrm{MeV} / \mathrm{amu}$ together with the present value at $150 \mathrm{MeV} / \mathrm{amu}$ are collected in fig. $3 \mathrm{a}$. The theoretical values calculated according to Bohr and Gillespie approximations are included for comparison. Figure 3b shows the same data in the collision strength representation. None of the theories used reproduces the data in a satisfactory way; the deviation of the Gillespie approximation, though suggestive, is not very significant for this light target.

\subsection{Non-radiative electron capture}

The earlier experiments determining the total capture cross sections for $\mathrm{He}^{++}$ions have been done $[8,9]$ in the energy range $17.3-43.3 \mathrm{MeV} / \mathrm{amu}$. At these low energies the capture is strongly dominated by the non-radiative process and the radiative electron capture can be neglected. Figure 4 presents these data together with the present value for $\sigma_{N R E C}$ at $150 \mathrm{MeV} / \mathrm{amu}$ as a function of the projectile velocity. The data are compared with theoretical calculations. The predictions of Nikolaev underestimate the effect dramatically. The discrepancy increases with velocity. The second order Oppenheimer-BrinkmannKrammer (B2) approximation [1] yields values close to 


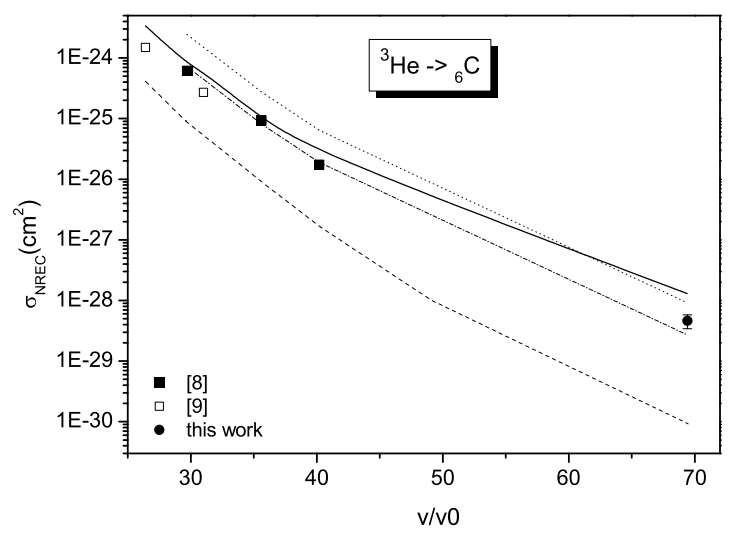

Fig. 4. The experimental (points) and calculated electron capture cross section as a function of projectile velocity. The solid line is from eikonal theory, dotted line is from OBK and dashdot line from B2 approximations, dashed line refers to Nikolaev formula.

the experiment though it slightly underestimates the effect at high velocity, while the refined eikonal theory deviates from the data in opposite direction with the velocity increasing.

\subsection{Radiative electron capture}

The radiative electron capture has never been measured for as light a projectile as helium. The present value can be compared with theoretical one calculated relativistically for all the shells: $\sigma_{R E C}=130 \mu \mathrm{b}$.

The non-relativistic analytical expression of Eichler [1] gives a lower value of $111 \mu \mathrm{b}$. The implicit assumption is that the electron in the carbon target can be considered as free and uncorrelated. The assumption seems to be plausible at the high helium velocity. It remains to be seen whether the rather large difference between the calculated and the experimental value can be related to the difference between the radiative recombination effect for $\mathrm{He}^{++}-\mathrm{C}$ and the REC effect for free electrons.

\section{Radiative electron capture by fast projectiles vs. the adiabaticity parameter}

The available systematics [15] of REC cross section for projectiles ranging from light ones up to as heavy as the hydrogen-like uranium shown in fig. 5 can be presented in the form of a universal curve as a function of the adiabaticity parameter:

$$
\eta=\left(\frac{v}{v_{e}}\right)^{2}
$$

where $v_{e}$ denotes the captured electron velocity in the projectile. The earlier data, obtained mainly for very heavy projectiles, correspond to $\eta<10$ and to $\sigma_{R E C}>1 \mathrm{~b}$. The present experiment extends this picture till $\eta=1500$

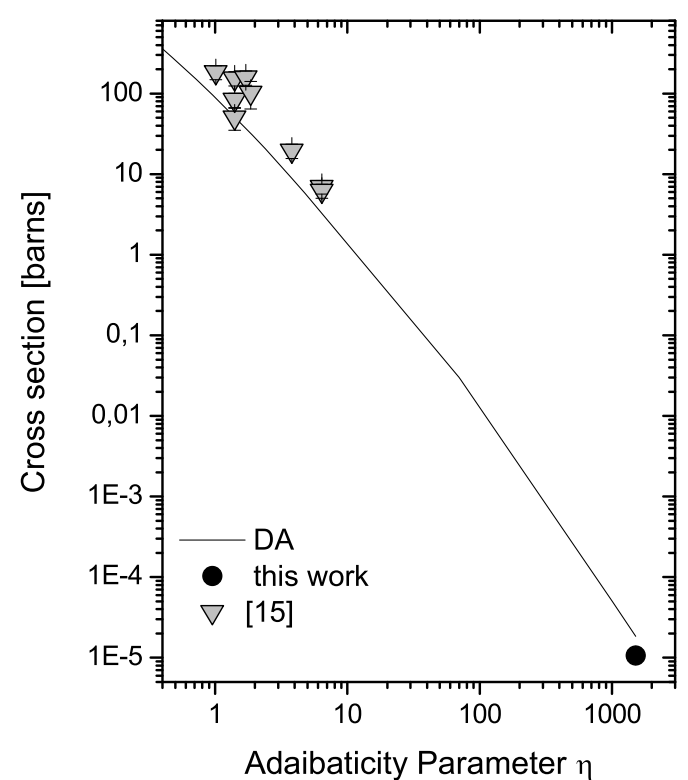

Fig. 5. The REC electron capture cross section per target electron measured for bare ions in collisions with light target atoms. The results are plotted as a function of the adiabaticity parameter and compared with the result of the Dipole Approximation (DA) theory.

and to the cross section several orders of magnitude lower, $\sigma_{R E C}=(64 \pm 12) \mu \mathrm{b}$. The theoretical value is about twice larger.

\section{Summary and conclusions}

Cross sections for the three main processes occurring for He ions traversing solid carbon have been determined at $150 \mathrm{MeV} / \mathrm{amu}$ bombarding energy. The processes in question are the stripping of electron from singly charged He ions, $\mathrm{He}^{+}$, and the radiative and non-radiative capture of electrons from the target to the vacant states in the $\mathrm{He}^{++}$ ions. This measurement, done at the highest bombarding energy ever used for such light ions, was feasible thanks to the use of advanced techniques of the modern nuclear physics. The results significantly extend the systematics of cross section versus energy data for all the three processes and permit to test the theoretical predictions in a sensitive region. Analysis of this systematics results in the following statements:

- There is no theory available to satisfactorily describe the energy dependence of the stripping cross section.

- The non-radiative electron capture from carbon to $\mathrm{He}^{++}$ions is more closely described by the relatively simple second order Oppenheimer-Brinkmann-Kramer approximation than by the more sophisticated eikonal theory. The latter seems to fail at high projectile velocities.

- The measured cross section for radiative transfer of the bound electron from the carbon target to the helium projectile is factor of two smaller than that calculated under the assumption of the REC process i.e. of the free 
electrons being captured in the time-reversed photoelectric effect. This is the first and the only measurement for radiative process, be it the Radiative Recombination or the Radiative Electron Capture, for such a light projectile at high adiabaticity parameter. Naively, the two processes should be practically identical at high energies. There might, however, be some constraints due to, e.g., the angular momentum coupling, which change the radiative recombination probability in comparison with the probability of free electron capture. The similarity of the binding energies in carbon atoms and in $\mathrm{He}^{+}$ions should also be noted. Clearly, there is a need for more studies of this difficult to measure, yet highly interesting effect.

The interaction of helium ions with free electrons is of primary astrophysical interest $[10,16]$. The REC photons due to radiative electron capture by bare $\mathrm{He}^{++}$ions may provide an observable for detecting these ions in the intergalactic space [17]. The fully ionized matter is otherwise unobservable by the usual optical methods. The present experiment yields the cross section value for this process in the observationally most interesting region of $E$ (electron) $\leq 100 \mathrm{keV}$.

\section{References}

1. J. Eichler, Phys. Rep. 193, 165 (1990).

2. N. Bohr, K. Dan, Vidensk. Selsk. Mat.-Fys. Medd. 18, No. 8 (1948).

3. G.H. Gillespie, Phys. Rev. A 18, 1967 (1978).

4. G.H. Gillespie, Phys. Rev. A 26, 2421 (1982).

5. K. Dennis et al., Phys. Rev. A 50, 3992 (1994).

6. V.S. Nikolaev, Zh. Eksp. Theor. Fiz. 51, 1263 (1966).

7. G. Lapicki et al., Phys. Rev. A 22, 1896 (1980).

8. I. Katayama et al., Phys. Lett. A 92, 385 (1982).

9. I. Katayama et al., Phys. Rev. A 53, 242 (1996).

10. Z. Sujkowski, Proceedings of the International Symposium on Advances in Nuclear Physics, Bucharest, Romania, 1999, edited by D. Poenaru, S. Stoica (World Scientific, 2000) p. 91.

11. D. Chmielewska, Proceedings of the International Winter Meeting on Nuclear Physics, Bormio, Italy, Suppl. No. 116 (2000) p. 90.

12. A. Gojska et al., Nucl. Instrum. Methods B 235, 368 (2005).

13. H. Akimune et al., Phys. Rev. C 52, 604 (1995).

14. E. Spindler et al., Phys. Rev. Lett. 42, 832 (1979).

15. Th. Stoehlker, Phys. Rev. A 51, 2098 (1995).

16. Z. Sujkowski, Nucl. Phys. A 719, 266c (2003).

17. D. Chmielewska, Z. Sujkowski, these proceedings. 